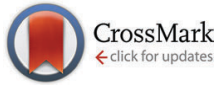

Cite this: Phys. Chem. Chem. Phys., $2015,17,32574$

DOI: $10.1039 / \mathrm{c} 5 \mathrm{cp} 90213 d$

www.rsc.org/pccp

\section{Correction: Size and shape dependent photoluminescence and excited state decay rates of diamondoids}

\author{
Robert Richter, ${ }^{* a}$ David Wolter, ${ }^{a}$ Tobias Zimmermann, ${ }^{a}$ Lasse Landt, ${ }^{a}$ \\ Andre Knecht, ${ }^{a}$ Christoph Heidrich, ${ }^{a}$ Andrea Merli, ${ }^{a}$ Otto Dopfer, ${ }^{a}$ Philipp Reiß, ${ }^{b}$ \\ Arno Ehresmann, ${ }^{b}$ Jens Petersen, ${ }^{c}$ Jeremy E. Dahl, ${ }^{d}$ Robert M. K. Carlson, ${ }^{d}$ \\ Christoph Bostedt, ${ }^{e}$ Thomas Möller, ${ }^{a}$ Roland Mitric ${ }^{c}$ and Torbjörn Rander*a
}

Correction for 'Size and shape dependent photoluminescence and excited state decay rates of diamondoids' by Robert Richter et al., Phys. Chem. Chem. Phys., 2014, 16, 3070-3076.

The authors would like to make the following correction to their article:

In Fig. 5, the second emission spectrum for the bottom (dia $\left.\left(D_{3 \mathrm{~d}}\right)\right)$ is shifted to higher energies and should be as seen in Fig. 1 below.

It has to be noted that by now, spectra with higher resolution are available. These can be found in ref. 1 .

The Royal Society of Chemistry apologises for these errors and any consequent inconvenience to authors and readers.

\title{
References
}

1 R. Richter, M. I. S. Röhr, T. Zimmermann, J. Petersen, C. Heidrich, R. Rahner, T. Möller, J. E. Dahl, R. M. K. Carlson, R. Mitric, T. Rander and A. Merli, Phys. Chem. Chem. Phys., 2015, 17, 4739.

\footnotetext{
${ }^{a}$ Institut für Optik und Atomare Physik, Technische Universität Berlin, Hardenbergstr. 36, 10623 Berlin, Germany. E-mail: robert.richter@tu-berlin.de

${ }^{b}$ Institute of Physics and Center for Interdisciplinary Nanostructure Science and Technology (CINSaT), University of Kassel, Heinrich-Plett-Str. 40, 34132 Kassel, Germany

${ }^{c}$ Universität Würzburg, Institut für Physikalische und Theoretische Chemie, Am Hubland, 97074 Würzburg, Germany

${ }^{d}$ Stanford Institute for Materials and Energy Sciences, Stanford University, Stanford, California 94305, USA

${ }^{e}$ SLAC National Accelerator Laboratory, Menlo Park, California 94025, USA
} 


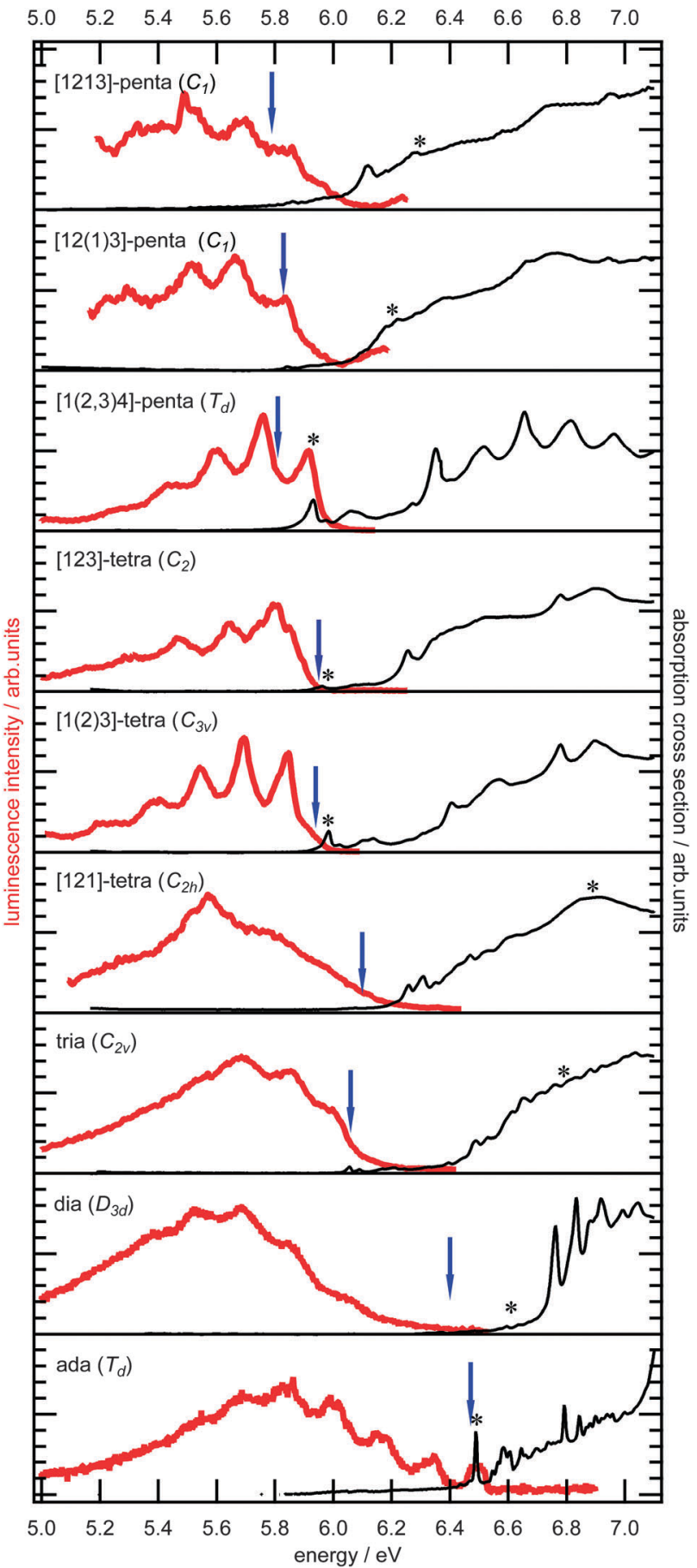

Fig. 1 Measured photoluminescence spectra (red) and absorption spectra (black) of selected isomers of the five smallest members of the diamondoid series. The excitation energy used to record the $\mathrm{PL}$ is marked with an asterisk and $E_{\text {gap }}$ is marked with a blue arrow. PL-spectra of adamantine ( $\Delta E=100$ $\mathrm{meV}$ ), diamantane $(\Delta E=100 \mathrm{meV})$, other spectra ( $\Delta E=25 \mathrm{meV}$, the spectra have been smoothed for presentation purposes). 